\title{
A new distance-regular graph of diameter 3 on 1024 vertices
}

\author{
Minjia Shi ${ }^{1}$ - Denis S. Krotov ${ }^{2}$ (D) Patrick Solé $^{3}$ (D) \\ Received: 26 June 2018 / Revised: 26 October 2018 / Accepted: 10 January 2019 / \\ Published online: 24 January 2019 \\ (c) The Author(s) 2019, corrected publication 2019
}

\begin{abstract}
The dodecacode is a nonlinear additive quaternary code of length 12 . By puncturing it at any of the twelve coordinates, we obtain a uniformly packed code of distance 5. In particular, this latter code is completely regular but not completely transitive. Its coset graph is distanceregular of diameter three on $2^{10}$ vertices, with new intersection array $\{33,30,15 ; 1,2,15\}$. The automorphism groups of the code, and of the graph, are determined. Connecting the vertices at distance two gives a strongly regular graph of (previously known) parameters $\left(2^{10}, 495,238,240\right)$. Another strongly regular graph with the same parameters is constructed on the codewords of the dual code. A non trivial completely regular binary code of length 33 is constructed.
\end{abstract}

Keywords Distance-regular graphs · Completely regular codes · Uniformly packed codes · Additive quaternary codes

Mathematics Subject Classification 05E30 · 94B05

\section{Introduction}

Distance-regular (DR) graphs form the most extensively studied class of structured graphs due to their many connections with codes, designs, groups and orthogonal polynomials

Communicated by J. H. Koolen.

This research is supported by National Natural Science Foundation of China (61672036), Excellent Youth Foundation of Natural Science Foundation of Anhui Province (1808085J20).

$凶 \quad$ Minjia Shi

smjwcl.good@163.com

Denis S. Krotov

krotov@math.nsc.ru

Patrick Solé

sole@enst.fr

1 School of Mathematical Sciences, Anhui University, Hefei 230601, Anhui, China

2 Sobolev Institute of Mathematics, pr. Akademika Koptyuga 4, Novosibirsk, Russia 630090

3 4CNRS/LAGA, University of Paris 8, 2 rue de la Liberté, 93526 Saint-Denis, France 
$[1,7]$. Since the times of Delsarte [13], a powerful way to create DR graphs, especially in low diameters, has been to use the coset graph of completely regular codes. A code is completely regular if the weight distribution of each coset solely depends on the weight of its coset leader. In [7] can be found many such examples from Golay codes, Kasami codes, and others. A recent survey is [4]. A special class of completely regular codes is that of uniformly packed codes. A code with packing radius $e$ is uniformly packed if the spheres of radius $e+1$ about the codewords overlap in a very uniform way: there are two constants $\lambda$ and $\mu$ such that the number of codewords at distance $e+1$ from some $x$ in ambient space is either $\lambda$ if $x$ is at distance $e$ from the code or $\mu$ if $x$ is at distance $\geq e+1$ from the code. In 1974 the following hypothetical parameters $[n, k, \geq 2 e+1]$ for quaternary uniformly packed codes with packing constants $(\lambda, \mu)$ were found [2] by computer search as

$$
n=\frac{2^{2 m+1}+1}{3}, \quad n-k=2 m+1, \quad e=2, \quad \mu=\lambda+1=\frac{2^{2 m}-1}{3}
$$

for $m \geq 2$, but no corresponding code was found. The case $m=2$ of the above parameters leads to a putative coset graph listed in [7, p.428] as a distance-regular graph on $1024=4^{5}$ vertices of diameter 3. It does not appear to be solved as of 2016 in the recent survey [23]. In 1998, an additive quaternary code of parameters $\left(12,4^{6}, 6\right)$ was introduced in [11] for the purpose of quantum-error correction, and called the dodecacode. This code was used in [17] to construct designs by shortening. In this note, we construct an additive non-linear code with the Bassalygo et al. parameters for $m=2$ by puncturing the dodecacode. From the coset graph of that code, we obtain a first known distance-regular graph with intersection array $\{33,30,15 ; 1,2,15\}$, already studied in [20], as a hypothetical object, from the standpoint of symmetry. This is the main result of this note. As a byproduct, connecting the vertices at distance two gives a strongly regular graph of parameters $\left(2^{10}, 495,238,240\right)$. Surprisingly, connecting the codewords in the dual code at distance 8 gives also a strongly regular graph with the same parameters. Other results coming from quaternary additive codes are as follows. By triple puncturing of the dodecacode, we obtain an additive code of length 9, and taking its coset graph, we construct a strongly regular graph of parameters $(64,27,10,12)$. We give a universal correspondence between quaternary and binary codes that preserves coset graphs, up to isomorphism. When applied to the two preceding codes, it constructs new completely regular binary codes in length 27 and 33 .

Thus, the observation that the punctured dodecacode is uniformly packed solves a problem that had been open since 1974 for quaternary codes and since 1989 for distance-regular graphs.

The sections are arranged as follows. The next section collects the necessary notation and definitions. Section 3 studies codes either quaternary or binary. Section 4 constructs the new distance regular graph announced in the title. Section 5 concludes the article and points out some challenging open problems.

As finding a new distance-regular graph is a most exciting result of the current work, we give here one of its shortest descriptions: it is the Cayley graph on $\mathbb{Z}_{2}^{10}$ with the connecting set $\{1,2,4,8,16,32,54,64,128,149,151,170,186,216,217,256,293,310,329,338$, $466,512,597,605,658,681,745,841,951,952,956,966,998\}$, where integers are treated as binary tuples in the standard way.

\section{Definitions and notation}

For any undefined term pertaining to codes we refer to [16,19], and to [1,7] for any undefined term related to distance-regular graphs. 


\subsection{Linear codes}

A linear code of length $n$, dimension $k$, minimum distance $d$ is called an $[n, k, d]$ code. A two-weight code is an $[n, k]$ linear binary code having two nonzero weights $w_{1}$ and $w_{2}$. Its parameters are denoted by the formula $\left[n, k ; w_{1}, w_{2}\right]$. The duality is understood with respect to the standard inner product. The external distance of a linear code is the number of nonzero weights in its dual. A coset of a linear code $C$ is any translate of $C$ by a constant vector. A coset leader is any coset element that minimizes the weight. The weight of a coset is the weight of any of its leaders. The coset graph $\Gamma_{C}$ of a code $C$ is defined on the cosets of $C$, two cosets being connected if they differ by a coset of weight one.

\subsection{Additive codes}

We consider codes of length $n$ for the Hamming distance over $\mathbb{F}_{4}$, and denote the Hamming weight of $x$ by $\operatorname{wt}(x)$. An additive code of length $n$ over $\mathbb{F}_{4}$ is an additive subgroup of $\mathbb{F}_{4}^{n}$. It is a free $\mathbb{F}_{2}$ module with $4^{k}$ elements for some $k \leq n$ (here $2 k$ is an integer, but $k$ may be half-integral). There are two ways to specify such a code $C$. Firstly, using a generator matrix $G$, the code $C$ can be cast as the $\mathbb{F}_{2}$-span of its rows. Secondly, a parity check matrix $H$ can be defined by using the trace inner product given in dimension 1 by $x * y=\operatorname{Tr}\left(x y^{2}\right)$, where $\operatorname{Tr}(z)=z+z^{2}$, and extended coordinatewise to vectors of length $n$. The dual of a code $C$ denoted by $C^{\perp}$ is understood with respect to that inner product. Define the $\star$-product of a $m \times n$ matrix $M$ with rows $M_{i}$ by a vector of length $n$ as

$$
M \star x=\left(M_{1} * x, \ldots, M_{m} * x\right)^{T},
$$

the ${ }^{T}$ denoting transposition. An additive code with $4^{k}$ codewords can then be specified by an $2 n-2 k$ by $n$ matrix $H$, the parity check matrix of $C$, as

$$
C=\left\{x \in \mathbb{F}_{4}^{n} \mid H \star x=0\right\} .
$$

Note that $H$ is a generator matrix for $C^{\perp}$. We call syndrome $s(x)$ of $x \in \mathbb{F}_{4}^{n}$ the column vector of size $2 n-2 k$ defined by $s(x)=H \star x$. A code $C^{-}$is obtained by puncturing from a code $C$ at the coordinate $i$ if it is the projection of $C$ on the remaining $n-1$ other coordinates. A coset of an additive code $C$ is any translate of $C$ by a constant vector. The definitions of coset leader and coset weight are the same as in the case of linear codes. The coset graph $\Gamma_{C}$ of a code $C$ is then the graph defined on the $4^{n-k}$ syndromes, two of them being connected if they differ by a syndrome $s(x)$ with $\operatorname{wt}(x)=1$. By the obvious one-to-one correspondence between syndromes and cosets [16], this graph is also the graph on the cosets, two cosets being connected if they differ by a coset of weight one. We give without proof the following extension of $[7,11.1 .11]$ from linear to additive codes.

Theorem 1 If $C$ is an additive quaternary code of minimum distance at least three, with dual weight distribution $\left[\left\langle i, A_{i}\right\rangle\right]$, then the spectrum of $\Gamma_{C}$ is $\left\{(3 n-4 i)^{A_{i}}\right\}$. Thus $A_{i}$ is the frequency of weight $i$ in $C^{\perp}$ and the multiplicity of the eigenvalue $3 n-4 i$.

The monomial automorphism group of a quaternary code $C$ is the set of all monomial transformations that leave the code wholly invariant, where a monomial transformation [16] is the composition of a coordinate permutation and the multiplication of the value in every position by a nonzero scalar. The complete automorphism group $\operatorname{Aut}(C)$ of a code $C$ is its stabilizer in the group of isometries of the Hamming space. The complete automorphism group includes the monomial automorphism group and the group of translations of the code, 
but can be larger than their product. An additive quaternary code is called completely transitive in the sense of [21] if the induced action of $\operatorname{Aut}(C)$ on the cosets of given weight of $C$ is transitive. Such codes are completely regular, with a distance-transitive coset graph. For an alternative definition see [14]. For a partial classification see [3].

\subsection{Graphs}

All graphs in this note are finite, undirected, connected, without loops or multiple edges. The neighborhood $\Gamma(x)$ is the set of vertices connected to $x$. The degree of a vertex $x$ is the size of $\Gamma(x)$. A graph is regular if every vertex has the same degree. The $i$-neighborhood $\Gamma_{i}(x)$ is the set of vertices at geodetic distance $i$ to $x$. A graph is distance regular (DR) if for every pair or vertices $u$ and $v$ at distance $i$ apart the quantities

$$
\begin{aligned}
a_{i} & =\left|\Gamma_{i+1}(u) \cap \Gamma(v)\right| \\
c_{i} & =\left|\Gamma_{i-1}(u) \cap \Gamma(v)\right|
\end{aligned}
$$

solely depend on $i$ and not on the special choice of the pair $(u, v)$. In that situation the graphs $\Gamma_{i}$ are regular of degree $v_{i}$ and we will refer to the $v_{i} \mathrm{~s}$ as the successive degrees of $\Gamma$. The automorphism group of a graph is the set of permutations of the vertices that preserve adjacency. A graph is distance-transitive if its automorphism group is transitive on its vertices and on each of the sets $\Gamma_{i}(x)$ for any $i$ and any fixed $x$. A DR graph of diameter 2 is called a Strongly Regular Graph (SRG). Its parameters $(\nu, \kappa, \lambda, \mu)$ denote the number of vertices, the degree, the number of common neighbors of a pair of connected vertices, the number of common neighbors of a pair of disconnected vertices. The spectrum of a graph is the set of distinct eigenvalues of its adjacency matrix. It is denoted by $\left\{\lambda_{1}^{m_{1}}, \lambda_{2}^{m_{2}}, \ldots\right\}$, where $m_{i}$ stands for the multiplicity of the eigenvalue $\lambda_{i}$. The Hamming graph $H(n, q)$ is the DR graph on $\mathbb{F}_{q}^{n}$, two vectors being connected if they are at Hamming distance one.

\section{Codes}

\subsection{Quaternary codes}

The dodecacode $D$ is a code of length 12 over $\mathbb{F}_{4}=\mathbb{F}_{2}(w)$ of parameters $\left(12,4^{6}, 6\right)$ that is additive but not linear [11]. It can be defined as a cyclic code with one generator. In the notation of [11] we have $D=(w 10100100101)$. Thus, puncturing at any of the twelve coordinates give an equivalent code $D^{-}$of parameters $\left(11,4^{6}, 5\right)$, of generator matrix given in Table 1.

Its primal and dual weight distributions and automorphism groups are easily computed in Magma [5] and Sage [22].

Theorem 2 The weight distribution and the dual weight distribution of $D^{-}$are

$$
\begin{gathered}
{[\langle 0,1\rangle,\langle 5,198\rangle,\langle 6,198\rangle,\langle 7,990\rangle,\langle 8,495\rangle,\langle 9,1650\rangle,\langle 10,330\rangle,\langle 11,234\rangle]} \\
\text { and }[\langle 0,1\rangle,\langle 6,198\rangle,\langle 8,495\rangle,\langle 10,330\rangle],
\end{gathered}
$$

respectively, where $\left\langle i, A_{i}\right\rangle$ means that there are $A_{i}$ codewords of weight $i$. 
Table 1 Generator matrix of $D^{-}$

$$
\left(\begin{array}{ccccccccccc}
0 & 0 & 0 & 0 & 0 & w^{2} & w^{2} & 0 & w & 1 & w \\
0 & 0 & 0 & 0 & 0 & w & 0 & w & w & w & 1 \\
1 & 0 & 0 & 0 & 0 & 1 & 0 & 1 & w^{2} & w^{2} & 1 \\
w & 0 & 0 & 0 & 0 & 0 & w & 1 & w & w & w \\
0 & 1 & 0 & 0 & 0 & 0 & 1 & 1 & 1 & w^{2} & w^{2} \\
0 & w & 0 & 0 & 0 & w^{2} & 1 & w^{2} & w & w & 0 \\
0 & 0 & 1 & 0 & 0 & w & w & 1 & 1 & 0 & 1 \\
0 & 0 & w & 0 & 0 & w & 1 & w & w^{2} & w^{2} & 0 \\
0 & 0 & 0 & 1 & 0 & w & w & w & 0 & 1 & w \\
0 & 0 & 0 & w & 0 & w^{2} & 1 & 1 & w^{2} & 0 & w \\
0 & 0 & 0 & 0 & 1 & w^{2} & w^{2} & w^{2} & 1 & 0 & w^{2} \\
0 & 0 & 0 & 0 & w & w & 1 & 0 & 1 & w & w
\end{array}\right)
$$

Theorem 3 The monomial automorphism group of $D^{-}$is generated by the following two monomial matrices:

$$
\begin{gathered}
{\left[\begin{array}{ccccccccccc}
0 & 0 & 0 & 0 & 0 & w & 0 & 0 & 0 & 0 & 0 \\
1 & 0 & 0 & 0 & 0 & 0 & 0 & 0 & 0 & 0 & 0 \\
0 & 0 & w^{2} & 0 & 0 & 0 & 0 & 0 & 0 & 0 & 0 \\
0 & 0 & 0 & 0 & 0 & 0 & 0 & w^{2} & 0 & 0 & 0 \\
0 & 0 & 0 & 0 & 0 & 0 & 0 & 0 & 0 & 1 & 0 \\
0 & 0 & 0 & 0 & w & 0 & 0 & 0 & 0 & 0 & 0 \\
0 & 0 & 0 & 0 & 0 & 0 & 0 & 0 & 0 & 0 & w \\
0 & 0 & 0 & 1 & 0 & 0 & 0 & 0 & 0 & 0 & 0 \\
0 & w^{2} & 0 & 0 & 0 & 0 & 0 & 0 & 0 & 0 & 0 \\
0 & 0 & 0 & 0 & 0 & 0 & 0 & 0 & w^{2} & 0 & 0 \\
0 & 0 & 0 & 0 & 0 & 0 & 1 & 0 & 0 & 0 & 0
\end{array}\right],} \\
{\left[\begin{array}{cccccccccccc}
1 & 0 & 0 & 0 & 0 & 0 & 0 & 0 & 0 & 0 & 0 \\
0 & 0 & 0 & 0 & 0 & 0 & 0 & 0 & 0 & 0 & w^{2} \\
0 & 0 & 0 & 0 & 0 & w^{2} & 0 & 0 & 0 & 0 & 0 \\
0 & 0 & 0 & 0 & 0 & 0 & 0 & w^{2} & 0 & 0 & 0 \\
0 & 0 & 0 & 0 & 0 & 0 & 0 & 0 & w & 0 & 0 \\
0 & 0 & w & 0 & 0 & 0 & 0 & 0 & 0 & 0 & 0 \\
0 & 0 & 0 & 0 & 0 & 0 & 0 & 0 & 0 & 1 & 0 \\
0 & 0 & 0 & w & 0 & 0 & 0 & 0 & 0 & 0 & 0 \\
0 & 0 & 0 & 0 & w^{2} & 0 & 0 & 0 & 0 & 0 & 0 \\
0 & 0 & 0 & 0 & 0 & 0 & 1 & 0 & 0 & 0 & 0 \\
0 & w & 0 & 0 & 0 & 0 & 0 & 0 & 0 & 0 & 0
\end{array}\right] .}
\end{gathered}
$$

It is isomorphic to a subgroup of $S_{33}$ of order $54=2 \times 3^{3}$ with generators $(142729$ 14 18)(2 5253015 16)(3 6262813 17)(7 9 8)(10 2212241123$)(193120322133)$ and (4 32)(5 33)(6 31)(7 17)(8 18)(9 16)(10 23)(11 24)(12 22)(13, 27)(14 25)(15 26)(19 28)(20 29)(21 30) in disjoint cycles product notation (this subgroup reflects the action of the automorphism group on the words of weight 1 in the order $10 \ldots 0, w 0 \ldots 0, w^{2} 0 \ldots 0$, 
$\left.010 \ldots 0, \ldots, 0 \ldots 0 w^{2}\right)$. The complete automorphism group of $D^{-}$is the product of the monomial automorphism group with the group of translations and has the order $54 \cdot 2^{12}$.

Remark 1 Since 54 is not divisible by 33 the code $D^{-}$cannot be completely transitive [21].

To compute the parameters $\lambda$ and $\mu$, we require the following lemma, of independent interest.

Lemma 1 If $C$ is a uniformly packed code of distance $2 e+1$, length $n$, and weight distribution $A_{i}$ over $\mathbb{F}_{q}$, then we have

$$
\begin{aligned}
\lambda(q-1)^{e}\left(\begin{array}{l}
n \\
e
\end{array}\right) & =A_{2 e+1}\left(\begin{array}{c}
2 e+1 \\
e
\end{array}\right) A_{2 e+1}\left(\begin{array}{c}
2 e+1 \\
e
\end{array}\right)(e+1)(q-2)+A_{2 e+2}\left(\begin{array}{c}
2 e+2 \\
e+1
\end{array}\right) \\
& =(\lambda-\mu) A_{2 e+1}\left(\begin{array}{c}
2 e+1 \\
e
\end{array}\right)+(\mu-1)(q-1)^{e+1}\left(\begin{array}{c}
n \\
e+1
\end{array}\right) .
\end{aligned}
$$

Proof Double counting the number of pairs $(x, y)$ such that $\operatorname{wt}(x)=e, \operatorname{wt}(y)=2 e+1$, $\operatorname{wt}(y-x)=e+1, y \in C$ relates $\lambda$ and $A_{2 e+1}$. This yields the first relation.

Double counting the number of pairs $(x, y)$ such that $\operatorname{wt}(x)=e+1, \operatorname{wt}(y) \in\{2 e+$ $1,2 e+2\}, \operatorname{wt}(y-x)=e+1, y \in C$ (note that among the $(q-1)^{e+1}\left(\begin{array}{c}n \\ e+1\end{array}\right)$ words of weight $e+1$, exactly $A_{2 e+1}\left(\begin{array}{c}2 e+1 \\ e\end{array}\right)$ are at distance $e$ from the code, and the other are at distance $e+1$ from the code) relates $\lambda, \mu, A_{2 e+1}$, and $A_{2 e+2}$. This gives us the second relation.

We are now in a position to state and prove the main result of this subsection.

Theorem 4 The code $D^{-}$is uniformly packed with $(\lambda, \mu)=(4,5)$.

Proof We know the code is uniformly packed since its minimum distance equals twice its external distance minus one [7, Corollary 11.1.2]. To compute $\lambda$ and $\mu$ we specialize the above lemma to $q=4, n=11, e=2$, and $A_{5}=A_{6}=198$ that is known from Theorem 2 . The first relation yields $\lambda=4$. Reporting into the second gives $\mu=5$.

Denote by $D^{3-}$ any $\left(9,4^{6}\right)$ code obtained by puncturing $D^{-}$on any pair of coordinates. The dual of $D^{3-}$ is an additive two-weight code. The following result comes from an easy Magma computation [5].

Theorem 5 The dual weight distribution of $D^{3-}$ is $[\langle 0,1\rangle,\langle 6,36\rangle,\langle 8,27\rangle]$.

\subsection{Binary codes}

We aim to construct a binary code with a coset graph isomorphic to that of $D^{-}$. To that end, we define a universal correspondence between quaternary and binary codes that preserves coset graphs, up to isomorphism.

Define the concatenation map of a quaternary code of length $n$ with the zero sum code $R_{3}^{\perp}=\{000,011,110,101\}$ as follows. For binary scalars $a$ and $b$, let $\phi(a+b w)=(b, b+$ $a, a)$. Equivalently, $\phi(c)=\left(\operatorname{Tr}(c), \operatorname{Tr}(w c), \operatorname{Tr}\left(w^{2} c\right)\right)$. The action of $\phi$ is extended to the quaternary vectors coordinatewise.

Theorem 6 Assume that $Q$ is a quaternary additive code of length $n$ and $B=\phi\left(Q^{\perp}\right)^{\perp}$. The following assertions hold:

(i) if $Q$ is an $\left(n, 4^{k}\right)$ code, then $B$ is a linear $[3 n, n+2 k]$ binary code; 
(ii) if $w$ is a weight of $Q^{\perp}$ with frequency $A_{w}$, then $2 w$ is a weight of $B^{\perp}$ with frequency $A_{w}$, and all the weights of $B^{\perp}$ arise in this way;

(iii) the coset graphs $\Gamma_{Q}$ and $\Gamma_{B}$ are isomorphic.

Proof (i) Trivially, the length of $B^{\perp}=\phi\left(Q^{\perp}\right)$ is $3 n$ and the size is the same as the size of $Q^{\perp}$, i.e., $4^{n-k}$. Hence, the size of $B$ is $2^{3 n} / 4^{n-k}=2^{n+2 k}$.

(ii) From the concatenation, we readily see that $\mathrm{wt}(\phi(c))=2 \mathrm{wt}(c)$.

(iii) We will show that the graphs $\Gamma_{Q}$ and $\Gamma_{B}$ built on the syndromes of the check matrices $P$ and $\phi(P)$ coincide. To see this, we first note that the both graphs are built on the binary columns of height $2 n-2 k$. Next, we consider the connecting syndromes that correspond to the weight- 1 vectors. Denote by $P_{1}$ the first column of the check matrix. The syndromes corresponding to the weight- 1 quaternary vectors $(1,0, \ldots, 0)$, $(w, 0, \ldots, 0)$, and $\left(w^{2}, 0, \ldots, 0\right)$ are $\operatorname{Tr}\left(P_{1}\right), \operatorname{Tr}\left(w^{2} P_{1}\right)$, and $\operatorname{Tr}\left(w P_{1}\right)$, where the trace map acts on the column component-wise. On the other hand, by the definition of $\phi$, the first three columns of $\phi(P)$ are $\operatorname{Tr}\left(P_{1}\right), \operatorname{Tr}\left(w P_{1}\right)$, and $\operatorname{Tr}\left(w^{2} P_{1}\right)$. Therefore, the syndromes corresponding to the binary 1 -weight vectors $(1,0, \ldots, 0),(0,1,0, \ldots, 0)$, $(0,0,1,0, \ldots, 0)$ are $\operatorname{Tr}\left(P_{1}\right), \operatorname{Tr}\left(w P_{1}\right), \operatorname{Tr}\left(w^{2} P_{1}\right)$ again. Considering in a similar way every other column of $P$, we find that the set of syndromes corresponding to the weight-1 vectors is the same for $P$ and $\phi(P)$. Hence, $\Gamma_{Q}=\Gamma_{B}$.

Remark 2 It can be shown that $Q$ and $B$ are related by the following correspondence (denoted by $\psi$ ) that associates $2^{n}$ codewords of $B$ to any codeword of $Q$. For simplicity, we write it for $n=1$.

$$
0 \stackrel{\psi}{\longrightarrow} 000,111 ; \quad 1 \stackrel{\psi}{\longrightarrow} 100,011 ; \quad w \stackrel{\psi}{\longrightarrow} 001,110 ; \quad w^{2} \stackrel{\psi}{\longrightarrow} 010,101 .
$$

Thus the images of any of the four symbols form an antipodal pair of vertices in the 3-cube. We have the following commutative diagram where the down arrow means "dual".

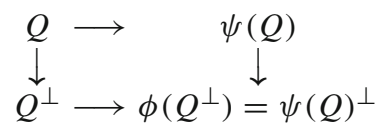

Thus $\psi$ is the pullback of $\phi$ in this diagram.

Corollary 1 Let $B^{-}=\phi\left(D^{-\perp}\right)^{\perp}$. The binary code $B^{-}$is a completely regular code of parameters [33, 23, 3], with dual weight distribution

$$
[\langle 0,1\rangle,\langle 12,198\rangle,\langle 16,495\rangle,\langle 20,330\rangle],
$$

and weight distribution

$$
\begin{aligned}
& {[\langle 0,1\rangle,\langle 3,11\rangle,\langle 5,198\rangle,\langle 6,1243\rangle,\langle 7,4158\rangle,\langle 8,13563\rangle,\langle 9,38445\rangle,} \\
& \langle 10,88638\rangle,\langle 11,185397\rangle,\langle 12,352902\rangle,\langle 13,568788\rangle,\langle 14,786885\rangle, \\
& \langle 15,998052\rangle,\langle 16,1156023\rangle,\langle 17,1156023\rangle,\langle 18,998052\rangle,\langle 19,786885\rangle, \\
& \langle 20,568788\rangle,\langle 21,352902\rangle,\langle 22,185397\rangle,\langle 23,88638\rangle,\langle 24,38445\rangle, \\
& \langle 25,13563\rangle,\langle 26,4158\rangle,\langle 27,1243\rangle,\langle 28,198\rangle,\langle 30,11\rangle,\langle 33,1\rangle] .
\end{aligned}
$$

Proof The dual weight distribution follows by the preceding theorem combined with Theorem

2. The weight distribution is then computed by MacWilliams transform. 
Remark 3 The code $B^{-}$is a non trivial example of a completely regular code since its minimum distance is 3 , and its external distance is 3 . Thus it is neither perfect, nor uniformly packed.

Corollary 2 The code $\phi\left(D^{3-\perp}\right)$ is a binary two-weight $[27,6 ; 12,16]$ code.

Proof The weight distribution follows by Theorem 6, where $n=9, k=6, Q=D^{3-}$, and $B=\phi\left(D^{3-\perp}\right)^{\perp}$, combined with Theorem 5 .

Remark 4 Binary completely regular codes with the last parameters are known [12]; all such codes are related to bent functions, see Theorem 12.12 and the following paragraph in [10]. Puncturing different coordinates, we obtain 3 nonequivalent codes with these parameters.

\section{Graphs}

In this section, we study the coset graphs of $D^{-}$, and of $D^{3-}$.

Theorem 7 The graph $\Gamma_{D^{-}}$is distance-regular of diameter 3, of spectrum

$$
\left\{27^{1}, 9^{198}, 1^{495},(-7)^{330}\right\} \text {. }
$$

Its successive degrees are $(1,33,495,495)$.

Proof The spectrum of $\Gamma_{D^{-}}$is easily computed from the weight distribution of Theorem 2 upon applying Theorem 1 . The distance-regularity follows from the fact that $D^{-}$is completely regular, being uniformly packed in the sense of [15], as having minimum distance $5=2 \times 3-1$ and external distance 3 . In particular it is completely regular as per [15, Theorem 7]. The fact that the error correcting capacity is 2 implies that the first three degrees are $1,33=$ $3 \times 11,495=\left(\begin{array}{c}11 \\ 2\end{array}\right) 3^{2}$. The last degree follows by $2^{10}-1-33-495=495$.

More structural information on this graph is as follows.

Theorem 8 The intersection array of the graph $\Gamma_{D^{-}}$is $\{33,30,15 ; 1,2,15\}$.

Proof We sketch the three steps of the proof as follows. We know $\lambda$ and $\mu$ from Theorem 4 . The outer distribution matrix $B$ of an uniformly packed code is uniquely determined by $n$, $e, q$ and these two parameters [7, Corollary 11.1.2]. From the known intersection array of $H(n, 4)$, and this data, the result follows by [7, Theorem 11.1.8], with $\Gamma=H(n, 4)$, and $\Pi$ being the completely regular partition induced by the cosets of $D^{-}$.

Theorem 9 The automorphism group of the graph has order $2^{10} \cdot 54$, acts transitively on the vertices, and has two orbits on the edges, of size $2^{9} \cdot 6$ and $2^{9} \cdot 27$. The stabilizer of a vertex has a structure of type $\left(C_{9} \rtimes C_{3}\right) \rtimes C_{2}$. The induced subgraph fixed by an element of the automorphism group, depending on the order $p$ of the element, can be the following, up to isomorphism:

(i) the whole graph, $p=1$;

(ii) the null graph, $p=2$;

(iii) the disjoint union of 8 complete graphs of order $4, p=2$;

(iv) the edge-free graph on 4 vertices, $p=3$;

(v) the Hamming graph $H(2,4), p=3$; 
(vi) the edge-free graph on 2 vertices, $p=6$;

(vii) the one-vertex graph, $p=9$;

(viii) the complete graph of order $4, p=9$.

Proof Since the graph is a Cayley graph, it is vertex transitive. The other assertions were established by Sage [22] computations.

The possible induced subgraphs fixed by an element of a prime order of the automorphism group of a distance-regular graph with intersection array $\{33,30,15 ; 1,2,15\}$ were studied in [20]. The case (iv) of the theorem above is missed in the main Theorem of [20], which should be completed by the subcase where every two vertices of the fixed subgraph are at distance three (A.A. Makhnev, Private Communication, 2018).

The following strongly regular graph is found by standard spectral techniques, as indicated in [7, p. 428]. For the next two results, we assume that the reader is familiar with the theory of duality in association schemes $[1,7,13]$.

Theorem 10 The graph $\left(\Gamma_{D^{-}}\right)_{2}$ is strongly regular with parameters $\left(2^{10}, 495,238,240\right)$.

Proof The $P$-matrix of the association scheme underlying $\Gamma_{D^{-}}$can be computed by the formulas in [9, pp. 135-136] or [13, Theorem 5.25], as

$$
\left(\begin{array}{cccc}
1 & 33 & 495 & 495 \\
1 & 9 & 15 & -25 \\
1 & 1 & -17 & 15 \\
1 & -7 & 15 & -9
\end{array}\right)
$$

The second column is the spectrum of $\left(\Gamma_{D^{-}}\right)_{2}$, discounting multiplicities. The result follows by the spectral characterization of SRGs [8].

Strongly regular graphs with these parameters are known [6]. We do not know if any of them is isomorphic to $\left(\Gamma_{D^{-}}\right)_{2}$.

There is a $Q$-analogue of the preceding results.

Theorem 11 The Delsarte dual of the underlying association scheme of $\Gamma_{D^{-}}$is $Q$-polynomial with Krein array $\{33,30,15 ; 1,2,15\}$, multiplicities 1, 33, 495, 495 and valencies 1 , $198,495,330$. The second relation of that association scheme is a SRG of parameters $\left(2^{10}, 495,238,240\right)$.

Proof Recall that the Delsarte dual of a coset graph is an association scheme on the codewords of the dual code [13], called hereafter the distance scheme. If the weights are numbered

$$
w_{1}=6<w_{2}=8<w_{3}=10,
$$

then for $x, y \in D^{-\perp}$, we define the relation $R_{i}$ as $x R_{i} y$ iff $\operatorname{wt}(x+y)=w_{i}$. By convention $x R_{0} y$ iff $x=y$. This is exactly the situation of [9] with $e=2$, up to the fact that $D^{-}$is not linear but only additive. By Delsarte duality, the multiplicities of this scheme are the degrees of $\Gamma_{D^{-}}$. Similarly, its valencies are the multiplicities of the spectrum of $\Gamma_{D^{-}}$. The Krein array coincides with the intersection array of the coset graph. The fact that the second relation of the distance scheme is a SRG comes from computation of the $P$-matrix which can be done as in [9, pp. 135-136]. 


$$
\left(\begin{array}{cccc}
1 & 198 & 495 & 330 \\
1 & 54 & 15 & -70 \\
1 & 6 & -17 & 10 \\
1 & -10 & 15 & -6
\end{array}\right)
$$

Remark 5 By Delsarte duality, the P-matrix of the coset scheme is the Q-matrix of the distance scheme and conversely.

Remark 6 This Q-polynomial scheme appears as an open problem in the table of Jason Williford [24]. We thank Bill Martin for pointing this out.

Remark 7 We do not know if the SRG constructed in that theorem is isomorphic with $\left(\Gamma_{D^{-}}\right)_{2}$.

We also obtain a strongly regular graph on the cosets of $D^{3-}$.

Theorem 12 The graph $\Gamma_{D^{3-}}$ is a SRG of parameters $(64,27,10,12)$. The spectrum of $\Gamma_{D^{3-}}$ is $\left\{27^{1}, 3^{36},(-5)^{27}\right\}$.

Proof The spectrum of $\Gamma_{D^{3-}}$ is easily computed from the weight distribution of Theorem 5 upon applying $[7,11.1 .11]$. The strong regularity follows then by the spectral characterization of SRG's [8]. The parameters follow by the data of the spectrum [8, Theorem 9.1.3].

Remark 8 Depending on the punctured coordinates we obtain 3 non-isomorphic SRG $(64,27,10,12)$. One of them is isomorphic to the SRG corresponding to a linear $[9,3]$ two-weight quaternary code. All the codes $D^{3-}$ are, however, nonlinear.

\section{Conclusion and open problems}

In this note, we have constructed the first additive non linear uniformly packed code in the history of the field. This solves a forty-four year old open problem of [2]. The other values of $m$ in the Introduction are worth investigating, even if they lead to graph parameters beyond the tables of $[7,23]$. The study of completely regular additive quaternary codes is only beginning.

On another tack, the existence of an uniformly packed code in a Doob graph of diameter 11 [7, p.27], a distance-regular graph with the same parameters as the $H(11,4)$, is a goal worth pursuing. Note that perfect codes are known in Doob graphs [18].

That object would constitute a Galois ring analogue of the (punctured) dodecacode, and might lead to another distance-regular graph with intersection array $\{33,30,15 ; 1,2,15\}$, non-isomorphic to the one considered in the current paper.

Acknowledgements We thank Jack Koolen, Alexander Makhnev, and Bill Martin for helpful discussions and the anonymous referees for useful comments.

Open Access This article is distributed under the terms of the Creative Commons Attribution 4.0 International License (http://creativecommons.org/licenses/by/4.0/), which permits unrestricted use, distribution, and reproduction in any medium, provided you give appropriate credit to the original author(s) and the source, provide a link to the Creative Commons license, and indicate if changes were made.

\section{References}

1. Bannai E., Ito T.: Algebraic Combinatorics I: Association Schemes. Benjamin/Cummings, Menlo Park (1984). 
2. Bassalygo L.A., Zaitsev G.V., Zinoviev V.A.: On uniformly packed codes. Probl. Inf. Transm. 10(1), 6-9 (1974). Translated from Probl. Peredachi Inf. 10(1), 9-14 (1974).

3. Borges J., Rifà J., Zinoviev V.A.: Nonexistence of completely transitive codes with error-correcting capability $e>3$. IEEE Trans. Inf. Theory 47(4), 1619-1621 (2001). https://doi.org/10.1109/18.923747.

4. Borges J., Rifà J., Zinoviev V.A.: Completely regular codes. arXiv:1703.08684 (2017).

5. Bosma W., Cannon J., Playoust C.: The Magma algebra system. I. The user language. J. Symb. Comput. 24(3-4), 235-265 (1997). https://doi.org/10.1006/jsco.1996.0125.

6. Brouwer A.E.: Parameters of strongly regular graphs. https://www.win.tue.nl/ aeb/graphs/srg/srgtab. html.

7. Brouwer A.E., Cohen A.M., Neumaier A.: Distance-Regular Graphs. Springer, Berlin (1989). https://doi. org/10.1007/978-3-642-74341-2.

8. Brouwer A.E., Haemers W.H.: Spectra of Graphs. Springer, New York (2012). https://doi.org/10.1007/ 978-1-4614-1939-6.

9. Calderbank A.R., Goethals J.M.: On a pair of dual subschemes of the Hamming scheme $H_{n}(q)$. Eur. J. Comb. 6(2), 133-147 (1985). https://doi.org/10.1016/S0195-6698(85)80004-4.

10. Calderbank R., Kantor W.M.: The geometry of two-weight codes. Bull. Lond. Math. Soc. 18(2), 97-122 (1986). https://doi.org/10.1112/blms/18.2.97.

11. Calderbank A.R., Rains E.M., Shor P.M., Sloane N.J.A.: Quantum error correction via codes over GF(4). IEEE Trans. Inf. Theory 44(4), 1369-1387 (1998). https://doi.org/10.1109/18.681315.

12. Koolen J., Krotov D., Martin B.: Completely regular codes: Tables, $\rho=2$. https://sites.google.com/site/ completelyregularcodes/h/r2

13. Delsarte P.: An algebraic approach to association schemes of coding theory. Philips Res. Rep. Suppl. 10, 1-97 (1973).

14. Giudici M., Praeger C.E.: Completely transitive codes in Hamming graphs. Eur. J. Comb. 20(7), 647-662 (1999). https://doi.org/10.1006/eujc.1999.0313.

15. Goethals J.M., van Tilborg H.C.A.: Uniformly packed codes. Philips Res. Rep. 30, 9-36 (1975).

16. Huffman W.C., Pless V.: Fundamentals of Error-Correcting Codes. Cambridge University Press, Cambridge (2003). https://doi.org/10.1017/CBO9780511807077.

17. Kim J.L., Pless V.: Designs in additive codes over GF(4). Des. Codes Cryptogr. 30(2), 187-199 (2003). https://doi.org/10.1023/A:1025484821641.

18. Krotov D.S.: Perfect codes in Doob graphs. Des. Codes Cryptogr. 80(1), 91-102 (2016). https://doi.org/ 10.1007/s10623-015-0066-6.

19. MacWilliams F.J., Sloane N.J.A.: The Theory of Error-Correcting Codes. North Holland, Amsterdam (1977).

20. Makhnev A.A.: On automorphisms of a distance-regular graph with intersection array $\{33,30,15 ; 1$, 2, 15\}. Dokl. Math. 90(3), 539-543 (2014). https://doi.org/10.1134/S1064562414070291.

21. Solé P.: Completely regular codes and completely transitive codes. Discret. Math. 81(2), 193-201 (1990). https://doi.org/10.1016/0012-365X(90)90152-8.

22. Stein W.A., et al.: Sage Mathematics Software, Version 6.9 (2015). http://www.sagemath.org.

23. van Dam E.R., Koolen J.H., Tanaka H.: Distance-regular graphs. Electron. J. Comb. Dyn. Survey \#DS22 (2016). http://www.combinatorics.org/ojs/index.php/eljc/article/view/DS22/pdf.

24. Williford J.S.: Table feasible parameter sets for primitive 3-class Q-polynomial association schemes. https://jaanos.github.io/tables/qpoly/qprim3_table.html.

Publisher's Note Springer Nature remains neutral with regard to jurisdictional claims in published maps and institutional affiliations. 